\title{
Knockdown of GA-binding protein subunit $\beta 1$ inhibits cell proliferation via p21 induction in renal cell carcinoma
}

\author{
SZU-CHIA CHEN ${ }^{1-4}$, MENG-CHI YEN ${ }^{5}$, FENG-WEI CHEN $^{1}$, LING-YU WU $^{1}$, \\ SHIANG-JIE YANG ${ }^{6}$, PO-LIN KUO ${ }^{1,7}$ and YA-LING HSU ${ }^{8}$ \\ ${ }^{1}$ Graduate Institute of Clinical Medicine, College of Medicine, Kaohsiung Medical University, Kaohsiung 807; \\ ${ }^{2}$ Division of Nephrology, Department of Internal Medicine; ${ }^{3}$ Department of Internal Medicine, \\ Kaohsiung Municipal Hsiao-Kang Hospital, Kaohsiung 812; ${ }^{4}$ Faculty of Medicine, College of Medicine, \\ Kaohsiung Medical University; ${ }^{5}$ Department of Emergency Medicine, Kaohsiung Medical University Hospital, \\ Kaohsiung Medical University; ${ }^{6}$ Department of Physical Medicine and Rehabilitation, School of Medicine, \\ College of Medicine, Kaohsiung Medical University, Kaohsiung 807; ${ }^{7}$ Institute of Medical Science and Technology, \\ National Sun Yat-Sen University, Kaohsiung $804 ;{ }^{8}$ Graduate Institute of Medicine, College of Medicine, \\ Kaohsiung Medical University, Kaohsiung 807, Taiwan, R.O.C.
}

Received January 18, 2018; Accepted May 4, 2018

DOI: $10.3892 /$ ijo.2018.4411

\begin{abstract}
Clear cell renal cell carcinoma (ccRCC) is the most common type of renal cancer. In the present study, bioinformatics tools were systematically used to investigate the potential upstream effector involved in the progression of ccRCC. Using the Gene Expression Omnibus database and Library of Integrated Network-based Cellular Signatures L1000 platform, it was identified that GA-binding protein subunit $\beta 1$ (GABPB1) was a potential effector gene. GABPB1 is a transcription factor subunit and its function in ccRCC is unclear. Elevated expression of GABPB1 mRNA in ccRCC was also observed in other clinical datasets from the Oncomine database. Following reverse transcription-quantitative polymerase chain reaction and western blot analysis, the ccRCC 786-O and A498 cell lines showed higher expression levels of GABPB1 than HK-2, a normal kidney cell line. Knockdown of GABPB1 in the 786-O and A498 cells significantly decreased the ability to form colonies by inducing the expression of $\mathrm{p} 21^{\text {Waf/Cip1 }}$. SurvExpress database analysis indicated that a higher expression of GABPB1 was associated with poor survival outcome in patients with renal cancer. These findings imply that GABPB1 serves an important role in the progression of ccRCC.
\end{abstract}

Correspondence to: Professor Ya-Ling Hsu, Graduate Institute of Medicine, College of Medicine, Kaohsiung Medical University, 100 Shih-Chuan 1st Road, Kaohsiung 807, Taiwan, R.O.C.

E-mail: hsuyl326@gmail.com

Key words: clear cell renal cell carcinoma, GA-binding protein subunit $\beta 1$, p2 $1^{\text {Waf/Cipl }}$ expression, oncogene, poor survival

\section{Introduction}

Renal cell carcinoma (RCC) is one of the 10 most common cancer types worldwide and the third most prevalent genitourinary cancer, accounting for $3 \%$ of all human malignancies (1). RCC is classified into different histopathological entities, with clear cell RCC (ccRCC) being the most common. Localized RCC remains a surgically treated disease, and $20-30 \%$ of patients who present with limited disease at the time of nephrectomy develop metastasis. Patients with advanced RCC have a low 5-year survival rate, with the time to relapse following nephrectomy ranging from 15 to 18 months (2). RCC is resistant to conventional cytotoxic drugs, and despite advances in immunotherapy and targeted therapies, a significant proportion of patients will experience disease recurrence and metastasis (3-5). There are currently few tumor biomarkers for RCC (6), and the identification of such biomarkers is important to allow for the early detection of RCC, to prioritize the asymptomatic individuals and to identify those at high-risk of progression $(7,8)$.

Transcription factors serve important roles in health and disease among the proteins regulating the development and maintenance of hematopoietic cells; they are essential for stem cell maintenance, proliferation and sufficient expansion of progenitors, and terminal differentiation of effector cells (9). The E26 transformation-specific (ETS) family of transcription factors are associated by conserved winged helix-loop-helix DNA-binding domains, and they have been shown to serve diverse roles in the development, differentiation and oncogenesis of cells (10). Of the $>24$ mammalian ETS factors identified to date, GA-binding protein (GABP) is the only obligate multimeric complex (11), and it is composed of two unrelated proteins, GABP subunit $\alpha$ (GABPA) and subunit $\beta 1$ (GABPB1). GABPA binds to DNA through its ETS domain, and GABPB1 contains the transcription activation domain (12). GABP has been associated with breast cancer (13), lung cancer (14), melanoma (15) 
and prostate cancer (16); however, the association between GABP and RCC is unclear. Therefore, the aim of the present study was to investigate the role of GABPB1 in RCC cell lines.

The study also aimed to identify vital upstream effectors in the tumorigenesis of ccRCC. The study approach was to systematically predict candidates using bioinformatics analysis tools and to validate the results in vitro. Combining the Gene Expression Omnibus (GEO) database and Library of Integrated Network-based Cellular Signatures (LINCS) L1000 platform, it was identified that GABPB1a was a potential effector gene. Reverse transcription-quantitative polymerase chain reaction (RT-qPCR) and western blot analysis were used to detect the expression of GABPB1 in the RCC cell lines, and the function of GABPB1 in ccRCC cell proliferation and survival of clinical renal tumors was investigated.

\section{Materials and methods}

Cell culture. A498, an RCC cell line, 786-O, a renal adenocarcinoma cell line, and HK-2, a cell line isolated from proximal tubule epithelial cells derived from normal kidneys and transformed with human papilloma virus 16 E6/E7 genes, were purchased from the American Type Culture Collection (Manassas, VA, USA). A498 and 786-O were grown in minimum essential media (Gibco; Thermo Fisher Scientific, Inc., Waltham, CA, USA) and RPMI-1640 (Lonza Group, Ltd., Basel, Switzerland), respectively. HK-2 was grown in keratinocyte serum-free medium (Gibco-BRL, Grand Island, $\mathrm{NY}$, USA) supplemented with $50 \mu \mathrm{g}$ bovine pituitary extract and $5 \mathrm{ng}$ recombinant epithelial growth factor per $\mathrm{ml}$ of basal medium. All media were supplemented with $10 \%$ fetal bovine serum (Gibco; Thermo Fisher Scientific, Inc.), and 10,000 units penicillin $/ 10 \mu \mathrm{g}$ streptomycin $/ 25 \mu \mathrm{g}$ amphotericin B per ml (Lonza Group, Ltd.). The cells were incubated at $37^{\circ} \mathrm{C}$ and in $5 \% \mathrm{CO}_{2}$. Trypsin-EDTA $(0.05 \%)$ (Gibco; Thermo Fisher Scientific, Inc.) was used for subculture.

Lenti-short hairpin (sh)RNA virus transduction. Lentivirus shRNAs were purchased from RNAi Core Facility (Taipei, Taiwan),including Lenti-shLuc976(cloneID,TRCN0000072249; targeting sequence: 5'-GCGGTTGCCAAGAGGTTCCAT-3') and two different clones of Lenti-shGABPB1 termed Lenti-shGABPB1\#1 (clone ID, TRCN0000019134; targeting sequence: 5'-GCAGAACCAAATCAACACAAA-3') and Lenti-shGABPB1\#2 (TRCN0000019138; targeting sequence: 5'-CCAGATGGACAACAAGTATTA-3'), respectively. The 786-O and A498 cell lines were incubated with complete media containing $8 \mu \mathrm{g} / \mathrm{ml}$ polybrene (EMD Millipore, Billerica, MA, USA) at $37^{\circ} \mathrm{C}$ for $30 \mathrm{~min}$. Lentiviruses (multiplicity of infection=3) were added for infection. After $24 \mathrm{~h}$ of incubation, the culture medium was refreshed with minimum essential media (A498) and RPMI-1640 (786-O), with $2 \mu \mathrm{g} / \mathrm{ml}$ puromycin (Sigma-Aldrich; Merck KGaA, Darmstadt, Germany), for $48 \mathrm{~h}$. The infected cells were then subcultured for 1 passage, incubated in medium with $2 \mu \mathrm{g} / \mathrm{ml}$ puromycin, and subsequently used in assays.

$R T-q P C R$. Cell lysates were homogenized and harvested with TRIzol reagent (Invitrogen; Thermo Fisher Scientific, Inc.). Total RNAs were extracted using Phenol:Chloroform:Isoamyl alcohol (25:24:1, v/v; Invitrogen; Thermo Fisher Scientific, Inc.) and isopropanol (Sigma-Aldrich; Merck $\mathrm{KGaA}$ ) according to the manufacturer's protocols. Total RNA concentration was determined according to the optical density (OD) at $260 \mathrm{~nm}$ using a PowerWave ${ }^{\mathrm{TM}}$ XS Microplate Spectrophotometer (BioTek Instruments, Inc., Winooski, VT, USA). RNAs (500 ng) were reverse transcribed into complementary DNAs using a PrimeScript $^{\mathrm{TM}}$ RT reagent kit (Takara Bio, Inc., Otsu, Japan) according to the manufacturer's instructions. qPCR was performed using Fast SYBR ${ }^{\circledR}$ Green Master mix and a StepOne Real-Time PCR system (both Applied Biosystems; Thermo Fisher Scientific, Inc.). The relative expression level was quantified using the $2^{-\Delta \Delta C q}$ method (17). All experiments were performed independently three times. The primers were as follows: GABPB1 forward, 5'-GGGGTCAGCAAGTCATC ACA-3' and reverse, 5'-GTCCATCTGGCATGGTCACA-3'; p21 $1^{\text {Waf/Cipl }}$ forward, 5'-CACCACTGGAGGGTGACTTC-3' and reverse, 5'-CGTGGGAAGGTAGAGCTTGG-3'; and glyceraldehyde 3-phosphate dehydrogenase (GAPDH) forward, 5'-GAGTCAACGGATTTGGTCGT-3' and reverse, 5'-TTGATT TTGGAGGGATCTCG-3'. The following thermocycling conditions were used: 1 cycle of $95^{\circ} \mathrm{C}$ for $20 \mathrm{sec}$, and 40 cycles of $95^{\circ} \mathrm{C}$ for $3 \mathrm{sec}$ and $60^{\circ} \mathrm{C}$ for $30 \mathrm{sec}$. GAPDH served as the internal control in this study.

Western blotting assay. Cell lysates were harvested in $1 \mathrm{X}$ radioimmunoprecipitation assay lysis buffer (EMD Millipore) with 1X protease inhibitor cocktail (Sigma-Aldrich; Merck KGaA). The clear liquid phase of total protein lysates was obtained by centrifugation at $14,500 \mathrm{xg}$ at $4^{\circ} \mathrm{C}$ for $15 \mathrm{~min}$. The protein concentration was determined using a BCA Protein assay kit (Novagen; EMD Millipore) and a PowerWave ${ }^{\mathrm{TM}}$ XS Microplate Spectrophotometer (BioTek Instruments, Inc.) according to the manufacturer's protocols. Protein lysates $(35 \mu \mathrm{g})$ were separated by $10 \%$ sodium dodecyl sulfate-polyacrylamide gel electrophoresis and transferred onto $0.45-\mu \mathrm{m}$ polyvinylidene difluoride membranes (Immobilon; EMD Millipore) pre-incubated in $100 \%$ methanol. The membranes were then incubated in blocking buffer $(5 \% \mathrm{w} / \mathrm{v}$ non-fat dried milk in $1 \mathrm{X}$ Tris-buffered saline with $0.2 \%$ Tween-20) at room temperature for $1 \mathrm{~h}$. The proteins were probed with diluted primary antibodies against GABPB1 (1:1,000; GTX103464; GeneTex, Inc.,Irvine, CA, USA), GAPDH (1:3,000; catalog no. 5174), cyclin A2 (1:1,000; catalog no. 4656), cyclin B1 (1:1,000; catalog no. 4138), cyclin D1 (1:1,000; catalog no. 2978), cyclin-dependent kinase (CDK)2 (1:1,000; catalog no. 2546), CDK4 (1:1,000; catalog no. 2906), CDK6 (1:1,000; catalog no. 3136) and p21 ${ }^{\text {Waf/Cipl } 1}(1: 1,000$; catalog no. 2946) (all from Cell Signaling Technology, Inc., Danvers, MA, USA) at $4^{\circ} \mathrm{C}$ overnight, and then hybridized with the specific secondary antibody, including peroxidase-conjugated goat anti-rabbit $\operatorname{IgG}(1: 3,000$; catalog no. AP132P) and peroxidase-conjugated goat anti-mouse $\operatorname{IgG}(1: 3,000$; catalog no. AP124P) (both from EMD Millipore) at room temperature for $1.5 \mathrm{~h}$. Subsequent to washing, chemiluminescent horseradish peroxidase substrate (Immobilon Western; EMD Millipore) was added. Signals were activated and detected using a FluorChem Imaging system (ProteinSimple, San Jose, CA, USA) according to the manufacturer's protocols. The intensity of protein signals was quantified using GelPro analyzer software (version 3.0; Media Cybernetics, Inc., Rockville, MD, USA). Each experiment was performed independently at least three times. 
Colony formation assay. The 786-O and A498 cell lines were transfected with Lenti-shLuc976, Lenti-shGABPB1\#1 and Lenti-shGABPB1\#2 and selected using $2 \mu \mathrm{g} / \mathrm{ml}$ puromycin for $48 \mathrm{~h}$. Subsequent to one passage, the cells were seeded onto a 6 -well plate at $5 \times 10^{2}$ cells/well and incubated at $37^{\circ} \mathrm{C}$ for 9 days Colonies in each well were stained with $0.4 \%$ crystal violet in $100 \%$ ethanol at room temperature for $1 \mathrm{~h}$ and counted. Each experiment was performed independently at least three times.

GEO database analysis. The GEO database is a webbased database providing meta information, including microarray, chip, RNA-seq and small RNA-seq of nextgeneration sequencing (NGS) data (https://www.ncbi.nlm.nih. gov/geo/) (18). Microarrays of GSE66271 and GSE66270 datasets on the microarray platform HG-U133_Plus_2 (Affymetrix Human Genome U133 Plus 2.0 Array; Affymetrix; Thermo Fisher Scientific, Inc.) (19) were selected, and the raw data were extracted using the GEO2R online tool (https://www. ncbi.nlm.nih.gov/geo/geo2r/) and re-plotted using GraphPad Prism5 software (GraphPad Software, La Jolla, CA, USA).

Oncomine database analysis. The Oncomine database integrates over 18,000 microarray experiments and 35 major cancer types (20). The raw data of GABPB1 mRNA expression compared in renal cancer and normal tissue (cancer vs. normal) were extracted from the 'Beroukhim' (21), 'Jones' (22), and 'Gumz' (23) datasets via the Oncomine database (http://www. oncomine.org) and re-plotted using GraphPad Prism5 software. The plot was illustrated with whiskers at the 10th and 90th percentiles. The criteria in the analysis were a P-value of $<0.05$, fold-change of $>1.2$ and a gene rank in the top $20 \%$. $\mathrm{P}$-values and gene differential expressions were calculated using the Oncomine database with two-sided Student's t-tests.

SurvExpress analysis. SurvExpress provides analysis of the effects of gene expression on the survival outcomes of different cancer types, as derived from microarray information from the The Cancer Genome Atlas (TCGA) database (https://tcgadata.nci.nih.gov). Survival curves comparing two populations at high and low risk of renal cancer were plotted using the SurvExpress online database (http://bioinformatica.mty.itesm. $\mathrm{mx}$ /SurvExpress). The samples of each dataset were split into two risk groups with the same size, for which each group was determined according to the ordered prognostic index (PI; high value denoting a high risk) (24). The raw data were extracted and re-plotted using GraphPad Prism5 software. The PI is the linear component of a Cox model, calculated as the gene expression value multiplied by values estimated from the Cox fitting (25).

LINCS L1000 platform. L1000 uses the ConnectivityMap database to provide gene expression signatures of 9 different cell lines treated with 16,425 perturbations of chemical molecules and drugs, of which 5,806 perturbations are knocked down and overexpressed single genes (26). Through querying the gene list of upregulated and downregulated genes, the results provided a perturbation list in which each perturbation caused a similar gene signature in cells with ranking scores.

Statistical analysis. The raw data was extracted and re-plotted by GraphPad Prism5 software (GraphPad Software). Student's t-test was used for statistical analysis in Figs. 2, 3 and 6. One-way analysis of variance was performed in Figs. 4 and 5. The Kaplan-Meier and log-rank test were adapted from SurvExpress website. $\mathrm{P}<0.05$ was considered to indicate a statistically significant difference.

\section{Results}

Identification of a potential upstream effector, GABPB1, in $c c R C C$. First, a microarray (GSE66271) was selected from the GEO database that provided mRNA expression profiles of 26 matched malignant renal tissues and adjacent normal renal tissues from 13 patients with ccRCC and metastasis. All malignant renal tumors had a ccRCC histology. The top 250 differentially expressed genes (103 upregulated and 134 downregulated genes, and 13 genes without gene symbols) were extracted using the GEO2R online tool. These differentially expressed genes were then used to predict upstream effectors using the L1000 platform. The 103 upregulated genes were input into the upregulated signature query and the 134 downregulated genes were input into the downregulated signature query, and then perturbations of the top 100 overexpressed genes in the analysis were selected. In contrast to this, the 134 downregulated genes were also input into the upregulated signature query and the 103 upregulated genes were input into the downregulated signature query, and perturbations of the top 100 knockdown genes in the analysis were selected. This approach attempted to identify upstream genes associated with the progression of renal cancer that are believed to be oncogenic and also upregulated in renal cancer. Following analysis of the combined results, 4 genes were identified: ATP synthase $\mathrm{H}^{+}$transporting mitochondrial Fo complex subunits (factor B) (ATP5S), solute carrier family 35 member F2 (SLC35F2), GABPB1 and transmembrane protein 110 (TMEM110) (Fig. 1). Of these genes, only the expression of GABPB1 was significantly upregulated in ccRCC tissues compared with that in adjacent normal tissues (Fig. 2A). There was no significant change in the expression of SLC35F2 (Fig. 2B), and the expression of ATP5S (Fig. 2C) and TMEM110 (Fig. 2D) was decreased in the ccRCC tissues compared with that in the normal tissues. The GSE66270 microarray provides mRNA expression profiles of 28 matched malignant renal tissues and adjacent normal renal tissues from 14 patients with ccRCC without metastasis. GABPB1 was also found to be significantly upregulated in these tumors (Fig. 2E).

GABPBI mRNA expression is increased in ccRCC. The Oncomine database was also used to analyze the mRNA expression of GABPB1 in different datasets comparing clinical renal tumors and normal renal tissues. A focus was placed on ccRCC, and the results from three datasets, including 'Beroukhim' renal non-hereditary (Fig. 3A) and hereditary (Fig. 3B), 'Jones' renal (Fig. 3C) and 'Gumz' renal (Fig. 3D), showed that the expression of GABPB1 was upregulated in ccRCC.

Knockdown of GABPB1 decreases colony formation ability in RCC cell lines. A higher expression level of GABPB1 was also observed in the RCC 786-O and A498 cell lines compared with that in the normal kidney HK-2 cell line. GABPB1 mRNA and 
Gene expression signature in ccRCC:

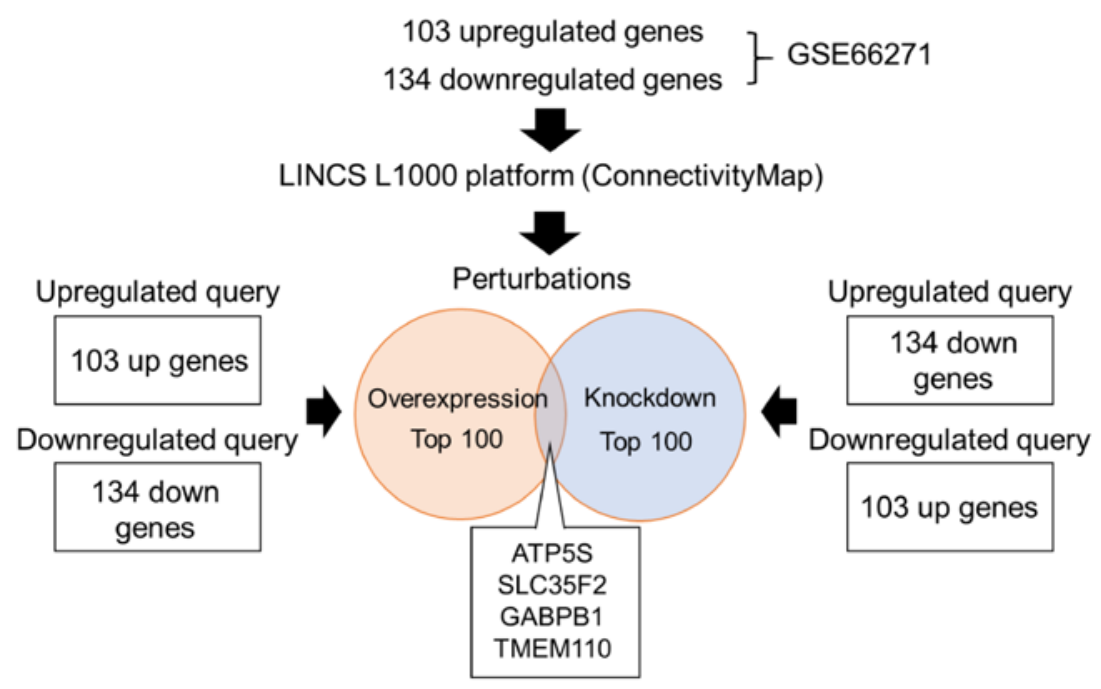

Figure 1. Flow chart of identification of upstream effectors by GEO database and LINCS L1000 platform. The GSE66271 microarray was selected from the GEO database. The differentially expressed genes (103 upregulated and 134 downregulated) were identified using the GEO2R web-tool. The LINCS L1000 platform was used to predict potential upstream effectors. GEO, Gene Expression Omnibus; LINCS, Library of Integrated Network-based Cellular Signatures; ccRCC, clear cell renal cell carcinoma.
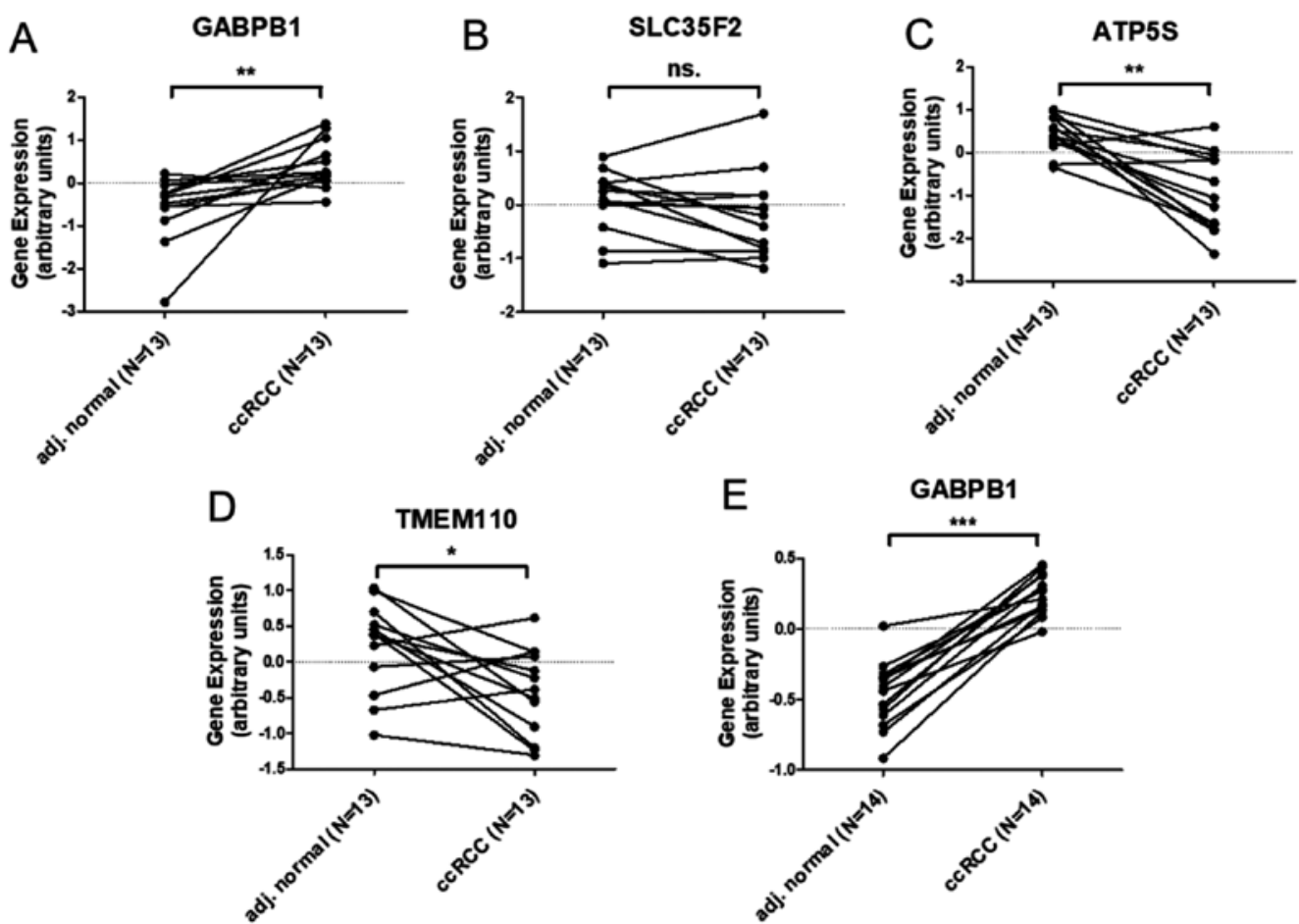

Figure 2. Analysis of gene expression in the GEO database. The gene expression of (A) GABPB1, (B) SLC35F2, (C) ATP5S and (D) TMEM110 was analyzed in the GSE66271 microarray, comparing adjacent normal tissues and ccRCC tissues (13 patients with metastatic diagnosis). (E) The gene expression of GABPB1 was analyzed in the GSE66270 microarray, comparing adjacent normal tissues and ccRCC tissues (14 patients without metastatic diagnosis). ${ }^{* * * *} \mathrm{P}<0.001$ and ${ }^{* *} \mathrm{P}<0.01$. GEO, Gene Expression Omnibus; ATP5S, ATP synthase $\mathrm{H}^{+}$transporting mitochondrial Fo complex subunits (factor B); SLC35F2, solute carrier family 35 member F2; GABPB1, GA-binding protein subunit $\beta 1$; ns, no significance; ccRCC, clear cell renal cell carcinoma; TMEM110, transmembrane protein 110 .

protein expression was upregulated in the $786-\mathrm{O}$ and $\mathrm{A} 498$ cells compared with that in the HK-2 cells (Fig. 4A and B). To study the role of GABPB1 in the progression of renal cancer, the RCC cell lines of 786-O and A498 were transfected with lentivirus-shRNA to knockdown GABPB1 (Fig. 4C and D). The results showed that knockdown of GABPB1 significantly decreased the colony formation ability in the 786-O and A498 cells (Fig. 4E).

Knockdown of GABPB1 inhibits cell growth via induction of p21 Waflicipl $m R N A$ expression. Compared with the shLuccontrol cells, 786-O and A498 cells exhibited significantly 

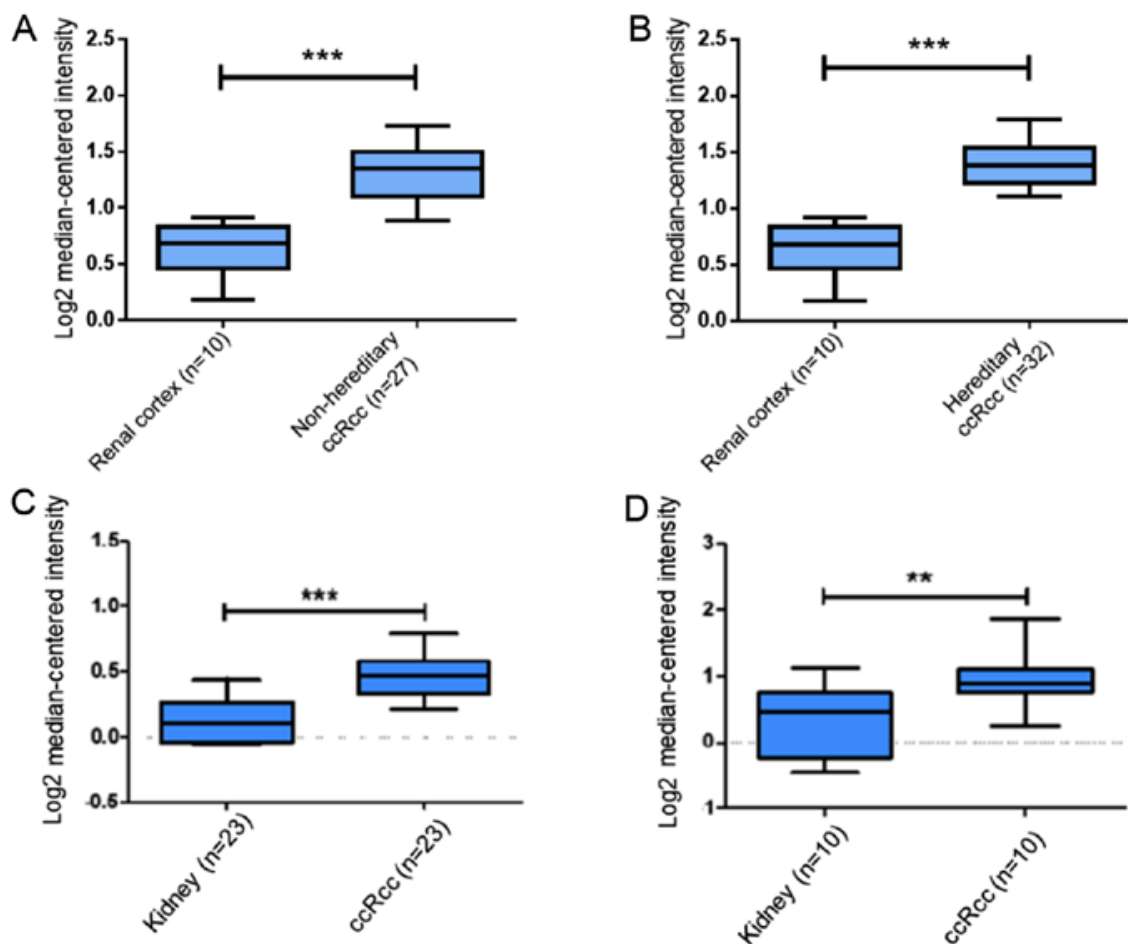

Figure 3. Analysis of GABPB1 gene expression in the Oncomine database. The mRNA expression of GABPB1 was analyzed in Oncomine datasets of (A) 'Beroukhim' renal - non-hereditary ccRCC vs. renal cortex (fold-change between renal cortex and non-hereditary ccRCC was 1.629) and (B) hereditary ccRCC vs. renal cortex (fold-change between renal cortex and non-hereditary ccRCC was 1.522), (C) 'Jones' renal - ccRCC vs. normal (fold-change between kidney and ccRCC was 1.253) and (D) 'Gumz' renal - ccRCC vs. normal (fold-change between kidney and ccRCC was 1.502). The raw data were extracted and re-plotted by GraphPad Prism5 software. Sample sizes are shown in parentheses. Student's t-test was used to evaluate the statistical difference between two groups. The criteria were $\mathrm{P}<0.05$, fold-change $>1.2$ and a gene rank in the top $20 \%$. ${ }^{* * *} \mathrm{P}<0.001$ and ${ }^{* *} \mathrm{P}<0.05$. ccRCC, clear cell renal cell carcinoma; GABPB1, GA-binding protein subunit $\beta 1$.
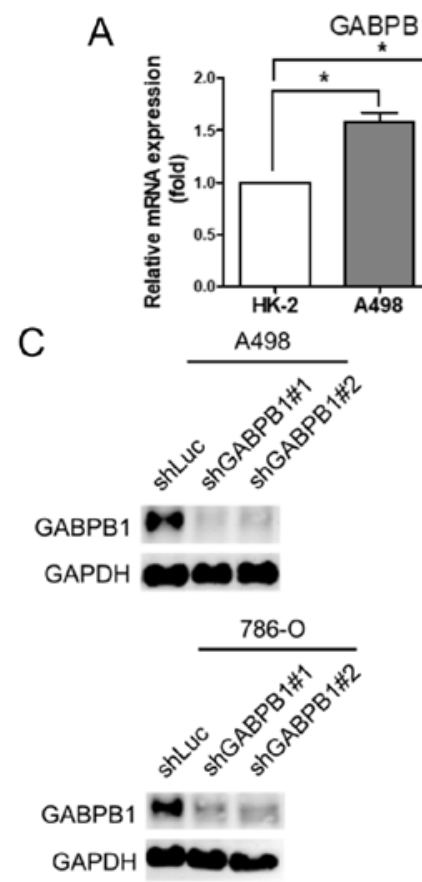

B

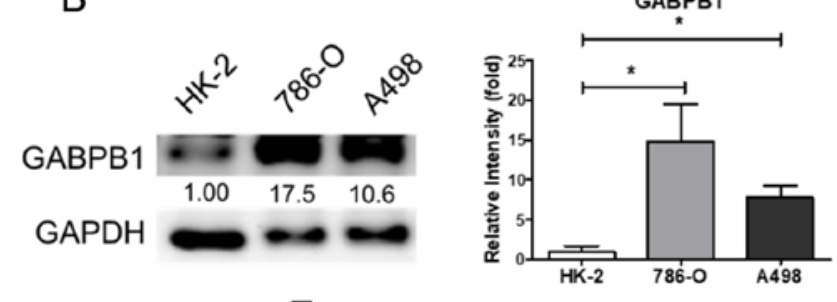

E
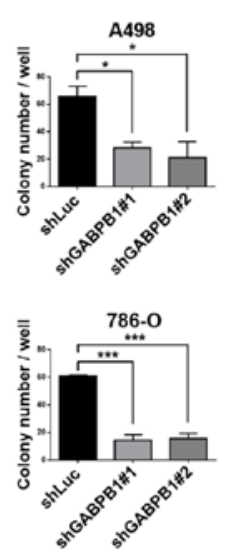

Figure 4. GABPB1 is increased in clear cell renal cell carcinoma cell lines and regulates cell proliferation. (A) The GABPB1 mRNA level was analyzed in HK-2, 786-O and A498 cell lines by reverse transcription-quantitative polymerase chain reaction. GAPDH was used as internal control. The relative expression level (normalized to GAPDH) was quantified by the $2^{-\Delta \triangle C q}$ method. (B) The GABPB1 protein expression was analyzed in HK-2, 786-O and A498 cell lines by western blotting. The bar plot indicates the relative quantification of each band intensity (normalized to GAPDH) compared with that of HK-2 cells. GABPB1-knockdown efficiency was determined at the (C) protein and (D) mRNA levels. (E) A colony formation assay was performed in cells transfected with lenti-shLuc, lenti-shGABPB1\#1 and lenti-shGABPB1\#2. Each experiment was completed with at least 3 independent replicates. ${ }^{* * *} \mathrm{P}<0.001,{ }^{* * *} \mathrm{P}<0.01$ and ${ }^{*} \mathrm{P}<0.05$. GABPB1, GA-binding protein subunit $\beta 1$; GAPDH, glyceraldehyde 3-phosphate dehydrogenase; sh, short hairpin. 
A
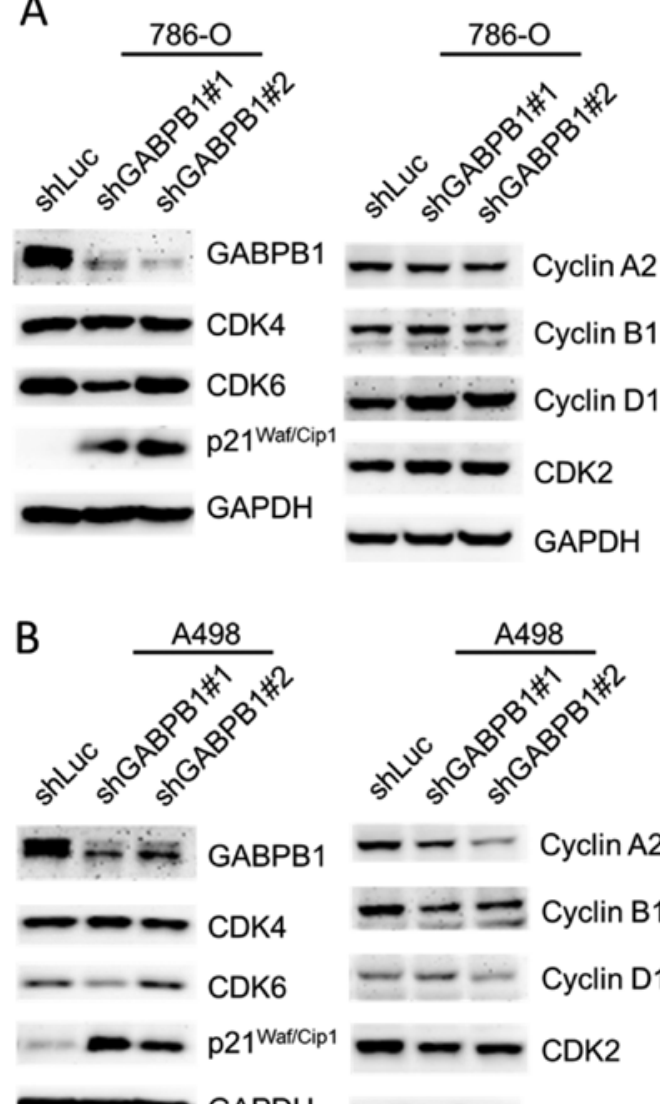

GAPDH
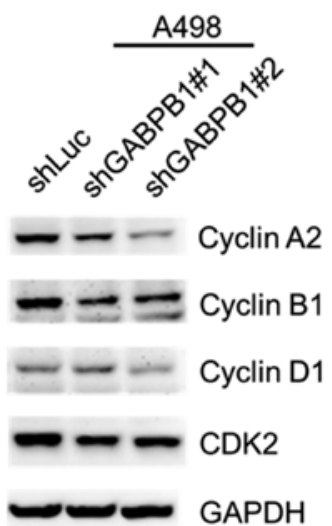

CDK2

GAPDH

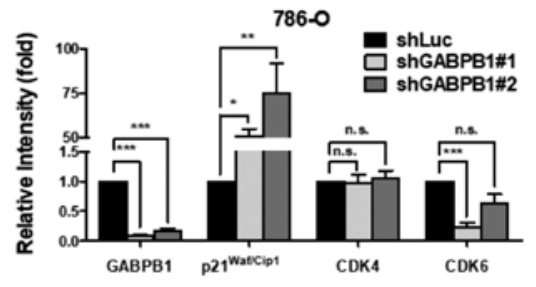

$786-0$
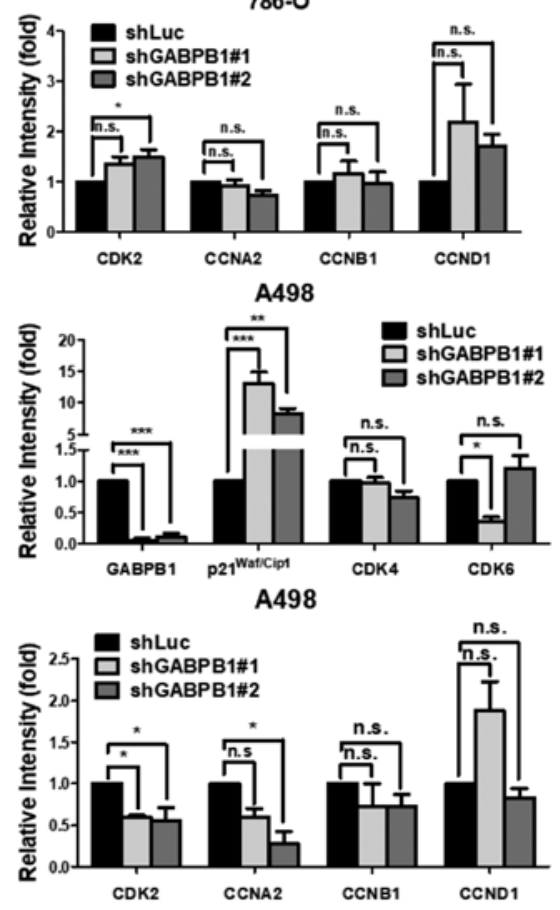

A498
C

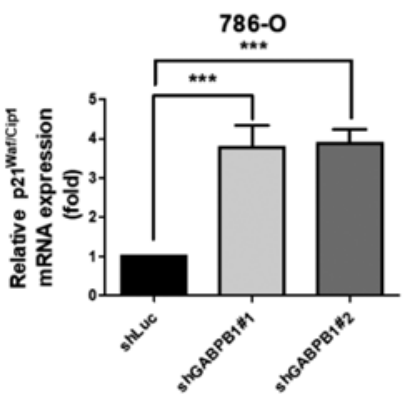

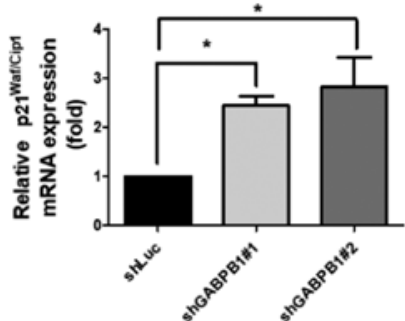

Figure 5. Knockdown of GABPB1 induces mRNA expression of $\mathrm{p} 21^{\text {Waf/Cip1 }}$. Western blotting analysis of total protein lysates from (A) 786-O and (B) A498 cells transduced with lenti-shLuc, lenti-shGABPB1\#1 and lenti-GABPB1\#2. The bar plots indicate the relative quantification of each band intensity (normalized to GAPDH) compared with that of shLuc. (C) The mRNA level of p21 ${ }^{\text {Waf/Cipl }}$ was analyzed in cells (786-O and A498) transfected with lenti-shLuc976, lenti-shGABPB1\#1 and lenti-GABPB1\#2. The relative expression was quantified by the $2^{-\Delta \Delta C q}$ method. Each experiment was completed with at least 3 independent replicates. ${ }^{* * * *} \mathrm{P}<0.001$, ${ }^{* *} \mathrm{P}<0.01$ and ${ }^{*} \mathrm{P}<0.05$. n.s., no significance; GABPB1, GA-binding protein subunit $\beta 1$; sh, short hairpinCDK, cyclin-dependent kinase; $\mathrm{CCN}$, cyclin.

increased $\mathrm{p} 21^{\text {Waf/Cip1 }}$ expression following knockdown of GABPB1 (Fig. 5A and B). In addition, the expression of CDK2, CDK6 and cyclin A2 was also affected; however, this change was not consistent between the 768-O and A498 cells, or when using shGABPB1\#1 and shGABPB1\#2, which targeted different sequences on GABPB1. As knockdown of GABPB1 via the two shGABPB1 resulted in an increased expression of $\mathrm{p} 21^{\text {Waf/Cip1 }}$, how the expression of $\mathrm{p} 21^{\text {Waf/Cip1 }}$ was regulated was then investigated. The results indicated that knockdown of GABPB1 increased the mRNA level of $\mathrm{p} 21^{\text {Waf/Cipl }}$ in the 786-O and A498 cells (Fig. 5C).

Increased expression of GABPBI is associated with poor survival outcomes in patients with renal cancer. The SurvExpress database was used to study the effects of the expression of GABPB1 on survival outcomes. There were three datasets, namely KIRC-TCGA Kidney renal clear cell carcinoma (Fig. 6A), KIPAN-TCGA Kidney PAN cancer TCGA June 2016 (Fig. 6B) and KIRP-TCGA Kidney renal papillary cell carcinoma June 2016 (Fig. 6C). The results showed that patients with renal cancer with poor survival outcomes had higher expression of GABPB1 compared with those with lower expression of GABPB1.

\section{Discussion}

The present study investigated vital upstream effectors involved in the tumorigenesis of ccRCC and identified a potential effector gene, GABPB1, using bioinformatics 

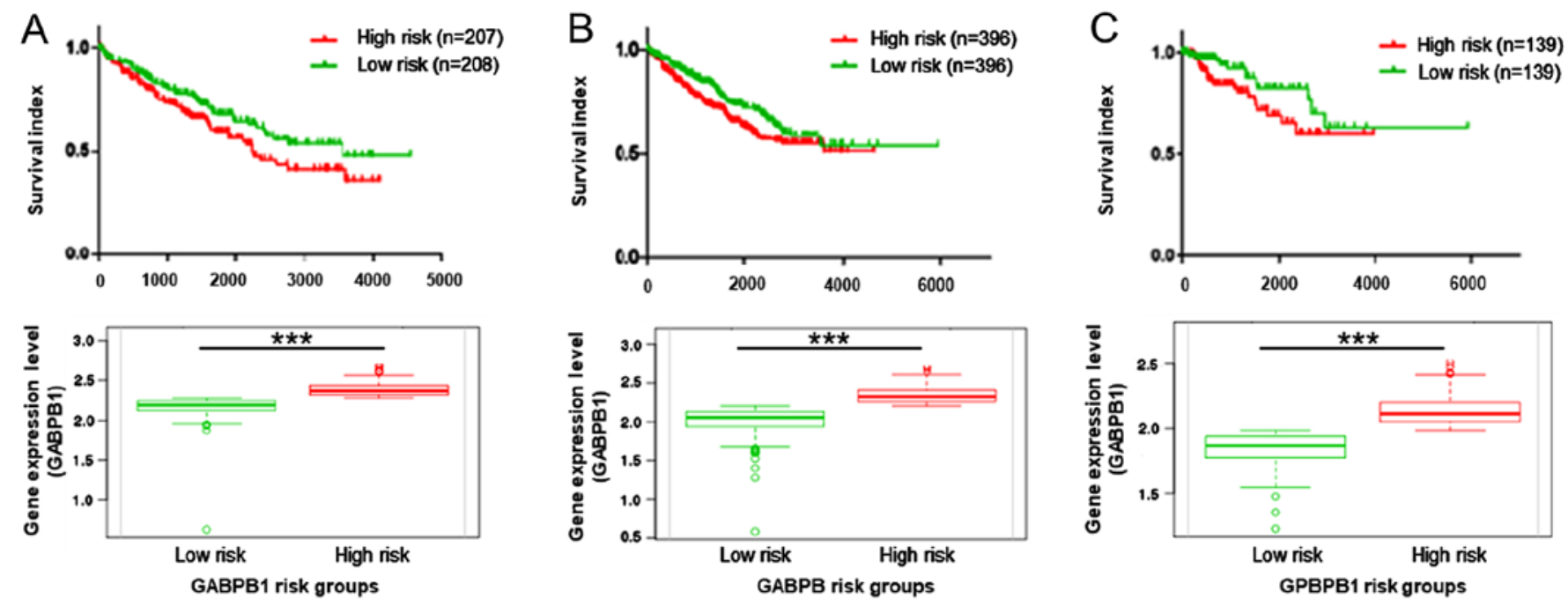

Figure 6. Analysis of effects of GABPB1 expression level in survival outcome of patients with renal cancer. The Kaplan-Meier curves comparing the high-risk population (red) and the low-risk population (green) of patients with renal cancer were created from data in the SurvExpress database. The threshold of $\mathrm{P}<0.05$ was determined. The box plots show the GABPB1 expression level in a corresponding population of each dataset. (A) KIRC-TCGA Kidney renal clear cell carcinoma (high risk, $\mathrm{n}=207$ and low risk, $\mathrm{n}=208 ; \mathrm{P}=0.04193$ ), (B) KIPAN-TCGA Kidney PAN cancer TCGA June 2016 (high risk, $\mathrm{n}=396$ and low risk, $\mathrm{n}=396$; $\mathrm{P}=0.02043$ ) and (C) KIRP-TCGA Kidney renal papillary cell carcinoma June 2016 (high risk, $\mathrm{n}=139$ and low risk, $\mathrm{n}=139 ; \mathrm{P}=0.02536$ ). The raw data of the survival curves were extracted and re-plotted using GraphPad Prism5 software. The expression level plots were captured from the SurvExpress web-database. ${ }^{* * *} \mathrm{P}<0.001$. GABPB1, GA-binding protein subunit $\beta 1$; TCGA, The Cancer Genome Atlas.

analysis and validated the results in vitro. A high expression of GABPB1 was observed in the RCC cell lines, and knockdown of GABPB1 significantly decreased the colony formation ability via induction of $\mathrm{p} 21^{\text {Waf/Cip1 }}$ expression. Furthermore, the patients at high risk of a poor survival outcome exhibited the highest expression level of GABPB1.

GABP has been reported to influence the development and lineage commitment in lymphoid and myeloid compartments (27-29), and also to promote chronic myeloid leukemia in mice (28). Manukjan et al (30) also reported that the expression of GABPA was positively correlated with the BCR-ABL1/ABL1 ratio in patients with chronic myeloid leukemia, and that it affected imatinib sensitivity in vitro. In addition, Yang et al (31) showed that genetic disruption of GABPA prevented entry into the $\mathrm{S}$ phase, and selectively reduced the expression of genes essential for DNA synthesis and the degradation of cyclin-dependent kinase inhibitors. Taken together, GABP appears to be necessary and sufficient to allow for re-entry into the cell cycle, and it appears to regulate a pathway distinct from that of D-type cyclins and cyclin-dependent kinases (31). In a hepatocellular carcinoma model, knockdown of GABP induced apoptosis and cell cycle arrest in the G0/G1 phase (32). In addition, reactivation of the expression of telomerase reverse transcriptase (TERT) was shown to enable cells to overcome replicative senescence and avoid apoptosis, which are fundamental steps in the initiation of human cancer $(33,34)$. Numerous types of cancer, including glioblastomas, have been shown to harbor highly recurrent TERT promoter mutations of unknown function (35). Bell et al (36) reported that the function of these mutations in glioblastomas was to recruit multimeric GABP transcription factors specifically to the mutant promoter, thereby directly linking TERT promoter mutations to the aberrant expression in a number of cancer types (36). In the present study, a higher expression level of GABPB1 was observed in the RCC cell lines, and knockdown of GABPB1 significantly decreased the colony formation ability. These findings suggest that GABPB1 may serve an important role in the progression of renal cancer.

To understand the mechanism by which GABPB1knockdown decreased cell growth, the expression levels of regulatory molecules involved in cell cycle progression were analyzed. It was found that compared with the shLuc-control cells, 786-O and A498 cells exhibited significantly increased $\mathrm{p} 21^{\text {Waf/Cipl } 1}$ expression following knockdown of GABPB1. Furthermore, knockdown of GABPB1 increased the mRNA levels of p21 ${ }^{\text {Waf/Cip1 }}$ in the $786-O$ and A498 cells. Progression of the cell cycle involves the activation of proteins, including cyclins and cyclin-dependent kinases, the catalytic subunits of cyclins. Cyclin-dependent kinase inhibitors can then bind to and inhibit the activity of cyclin/cyclin-dependent kinase complexes (37). One such inhibitor, p21 $1^{\text {Waf/Cip1 }}$, has been shown to bind to these complexes and inhibit their activity in response to DNA damage and anti-mitogenic or differentiating signals (38). Increasing evidence suggests that growth arrest along with the upregulation of p21 can inhibit proliferation and promote differentiation $(37,38)$. p21 has also been reported to inhibit DNA replication through inhibition of proliferating cell nuclear antigens, which is necessary for the progression of the cell cycle $(37,39)$. Using bioinformatics analysis, the present study identified a potential tumor-associated gene, GABPB1, in renal cancer, and also found that the knockdown of GABPB1 decreased cell growth via the induction of $\mathrm{p} 21^{\text {Waf/Cipl }}$ expression.

The regulation between GABPB1 and $21^{\text {waf/Cip1 }}$ was not clear in the present study. BRCA1 DNA repair-associated (BRCA1) is an important cell cycle regulator, and it may be a potential regulator in a GABPB1 and $\mathrm{p} 21^{\text {Waf/Cip1 }}$ axis. In addition, BRCA1 is a tumor suppressor that has been shown to maintain genomic stability and prevent cell transformation. Ritter et al (40) reported that glucocorticoid receptors interact 
with GABPB at the BRCA1 promoter, thereby inducing the expression of BRCA1, which then upregulates the expression of $\mathrm{p} 21^{\mathrm{Waf} / \mathrm{Cip} 1}$ in breast cancer. Another previous study reported that wild-type BRCA1 increased the expression of $\mathrm{p} 21^{\mathrm{Waf} / \mathrm{Cip} 1}(41)$. However, the present results revealed that silencing GABPB1 resulted in induction of $\mathrm{p} 21^{\mathrm{Waf} / \mathrm{Cip} 1}$ expression. This implies that BRCA1-mediated cell cycle regulation does not involve GABPB1 or $\mathrm{p} 21^{\mathrm{Waf} / \mathrm{Cip} 1}$ in renal cancer cells. The limitation of this study is that there was no direct evidence to support whether BRCA1 is involved in regulating GABPB1 and $\mathrm{p} 21^{\text {Waf/Cip1 }}$. Therefore, our future studies will investigate the detailed mechanism in renal cancer cell models and animal tumor models.

$\mathrm{RCC}$ is a malignancy arising from the renal tubule epithelium (42) and it is the most common type of kidney cancer in adults, accounting for $90-95 \%$ of cases. Initially, treatment involves partial or complete removal of the affected kidneys (43). Metastatic RCC most commonly involves spread to the bones, lungs, lymph nodes, brain, liver and adrenal glands. The median survival time of patients with metastatic RCC is $\sim 8$ months (44), and $50 \%$ of these patients will succumb within the first year, with only $10 \%$ surviving for $>5$ years (45). Invasion and spread to other organs are the greatest challenges to therapy (46). Degradation of the extracellular matrix and basement membrane, which are tissue barriers for tumor cells, serve a key role in tumor proliferation and metastasis (47). Heparanase is an enzyme that degrades heparan sulfate proteoglycans and is predominantly expressed in high-grade $\mathrm{RCC}$, and it has been shown to be positively associated with the pathological tumor stage and a poor prognosis (48). GABP is one of the transcription factors involved in regulating the expression of the heparanase-1 gene (49), and Rao et al (50) showed that B-Raf proto-oncogene serine/threonine kinase (BRAF)-knockdown suppressed GABPB expression and thereby regulated heparanase-1 promoter activity. Increased expression of heparanase-1 may therefore contribute to the aggressive behavior of BRAF-mutated cancer (50). The present study also showed that the high-risk population associated with poor survival exhibited higher expression levels of GABPB1, suggesting that GABPB1 serves a key prognostic role in renal cancer.

In conclusion, the significance of present study is that it revealed the crucial role of GABPB1, which has not been identified in previous studies, via combination of the L1000 platform and GEO database in renal cancer. Furthermore, a key regulator, p21 ${ }^{\text {Waf/Cip1 }}$, was determined for GABPB1-mediated oncogenic signaling. The detailed regulatory mechanism between GABPB1 and $\mathrm{p} 21^{\text {Waf/Cip1 }}$ requires further investigation in the future; however, the present study findings suggest that GABPB1 may be a potential tumor-associated gene in the progression of RCC.

\section{Acknowledgements}

Not applicable.

\section{Funding}

This study was supported by grants from the Ministry of Science and Technology (no. 104-2314-B-037-053-MY4) and the
Kaohsiung Medical University Hospital Research Foundation (no. KMUHS10601).

\section{Availability of data and materials}

The datasets used and/or analyzed during the current study are available from the corresponding author on reasonable request.

\section{Authors' contributions}

SC and YH designed the study; FC and LW performed the experiments for the study; MY, FC, SY, PK and YH analyzed the data and interpreted the results; and SC, MY and $\mathrm{YH}$ wrote the manuscript.

\section{Ethics approval and consent to participate}

Not applicable.

\section{Consent for publication}

Not applicable.

\section{Competing interests}

The authors declare that they have no competing interests.

\section{References}

1. DeSantis CE, Lin CC, Mariotto AB, Siegel RL, Stein KD, Kramer JL, Alteri R, Robbins AS and Jemal A: Cancer treatment and survivorship statistics, 2014. CA Cancer J Clin 64: 252-271, 2014.

2. Athar $U$ and Gentile TC: Treatment options for metastatic renal cell carcinoma: A review. Can J Urol 15: 3954-3966, 2008.

3. LaGory EL, Wu C, Taniguchi CM, Ding CC, Chi JT, von Eyben R, Scott DA, Richardson AD and Giaccia AJ: Suppression of PGC-1 $\alpha$ is critical for reprogramming oxidative metabolism in renal cell carcinoma. Cell Rep 12: 116-127, 2015.

4. Rini BI and Atkins MB: Resistance to targeted therapy in renalcell carcinoma. Lancet Oncol 10: 992-1000, 2009.

5. Rini BI, Campbell SC and Escudier B: Renal cell carcinoma. Lancet 373: 1119-1132, 2009.

6. Frank I, Blute ML, Cheville JC, Lohse CM, Weaver AL and Zincke H: An outcome prediction model for patients with clear cell renal cell carcinoma treated with radical nephrectomy based on tumor stage, size, grade and necrosis: The SSIGN score. J Urol 168: 2395-2400, 2002.

7. Seliger B, Dressler SP, Lichtenfels R and Kellner R: Candidate biomarkers in renal cell carcinoma. Proteomics 7: 4601-4612, 2007.

8. Xu Z, Yang F, Wei D, Liu B, Chen C, Bao Y, Wu Z, Wu D, Tan H, Li J, et al: Long noncoding RNA-SRLR elicits intrinsic sorafenib resistance via evoking IL-6/STAT3 axis in renal cell carcinoma. Oncogene 36: 1965-1977, 2017.

9. Orkin SH and Zon LI: Hematopoiesis: An evolving paradigm for stem cell biology. Cell 132: 631-644, 2008.

10. Hsu T, Trojanowska M and Watson DK: Ets proteins in biological control and cancer. J Cell Biochem 91: 896-903, 2004.

11. Rosmarin AG, Resendes KK, Yang Z, McMillan JN and Fleming SL: GA-binding protein transcription factor: A review of GABP as an integrator of intracellular signaling and protein-protein interactions. Blood Cells Mol Dis 32: 143-154, 2004.

12. LaMarco K, Thompson CC, Byers BP, Walton EM and McKnight SL: Identification of Ets- and notch-related subunits in GA binding protein. Science 253: 789-792, 1991.

13. Polansky H and Javaherian A: Commentary: The unliganded glucocorticoid receptor positively regulates the tumor suppressor gene BRCA1 through GABP beta. Front Cell Infect Microbiol 5: 66,2015 . 
14. El-Aarag SA, Mahmoud A, Hashem MH, Abd Elkader H, Hemeida AE and ElHefnawi M: In silico identification of potential key regulatory factors in smoking-induced lung cancer. BMC Med Genomics 10: 40, 2017.

15. Zhang T, Xu M, Makowski MM, Lee C, Kovacs M, Fang J, Willems E, Trent JM, Hayward NK, Vermeulen M, et al: SDHD promoter mutations ablate GABP transcription factor binding in melanoma. Cancer Res 77: 1649-1661, 2017.

16. Simmonds P, Loomis E and Curry E: DNA methylation-based chromatin compartments and ChIP-seq profiles reveal transcriptional drivers of prostate carcinogenesis. Genome Med 9: 54 2017.

17. Livak KJ and Schmittgen TD: Analysis of relative gene expression data using real-time quantitative PCR and the 2(-Delta Delta C(T)) method. Methods 25: 402-408, 2001.

18. Clough E and Barrett T: The Gene Expression Omnibus Database. Methods Mol Biol 1418: 93-110, 2016.

19. Wotschofsky Z, Gummlich L, Liep J, Stephan C, Kilic E, Jung K, Billaud JN and Meyer HA: Integrated microRNA and mRNA signature associated with the transition from the locally confined to the metastasized clear cell renal cell carcinoma exemplified by miR-146-5p. PLoS One 11: e0148746, 2016.

20. Rhodes DR, Yu J, Shanker K, Deshpande N, Varambally R, Ghosh D, Barrette T, Pandey A and Chinnaiyan AM: ONCOMINE: A cancer microarray database and integrated data-mining platform. Neoplasia 6: 1-6, 2004.

21. Beroukhim R, Brunet JP, Di Napoli A, Mertz KD, Seeley A, Pires MM, Linhart D, Worrell RA, Moch H, Rubin MA, et al: Patterns of gene expression and copy-number alterations in von-hippel lindau disease-associated and sporadic clear cell carcinoma of the kidney. Cancer Res 69: 4674-4681, 2009.

22. Jones J, Otu H, Spentzos D, Kolia S, Inan M, Beecken WD, Fellbaum C, Gu X, Joseph M, Pantuck AJ, et al: Gene signatures of progression and metastasis in renal cell cancer. Clin Cancer Res 11: 5730-5739, 2005

23. Gumz ML, Zou H, Kreinest PA, Childs AC, Belmonte LS, LeGrand SN, Wu KJ, Luxon BA, Sinha M, Parker AS, et al: Secreted frizzled-related protein 1 loss contributes to tumor phenotype of clear cell renal cell carcinoma. Clin Cancer Res 13 : 4740-4749, 2007.

24. Aguirre-Gamboa R, Gomez-Rueda H, Martínez-Ledesma E, Martínez-Torteya A, Chacolla-Huaringa R, RodriguezBarrientos A, Tamez-Peña JG and Treviño V: SurvExpress: An online biomarker validation tool and database for cancer gene expression data using survival analysis. PLoS One 8: e74250, 2013

25. Collett D: Modelling survival data in medical research. 3rd edition. CRC Press, Bristol, p548, 2015.

26. Duan Q,Flynn C,Niepel M,HafnerM,Muhlich JL,FernandezNF, Rouillard AD, Tan CM, Chen EY, Golub TR, et al: LINCS Canvas Browser: Interactive web app to query, browse and interrogate LINCS L1000 gene expression signatures. Nucleic Acids Res 42: W449-W460, 2014

27. Xue HH, Bollenbacher J, Rovella V, Tripuraneni R, Du YB, Liu CY, Williams A, McCoy JP and Leonard WJ: GA binding protein regulates interleukin 7 receptor alpha-chain gene expression in T cells. Nat Immunol 5: 1036-1044, 2004.

28. Xue HH, Bollenbacher-Reilley J, Wu Z, Spolski R, Jing X, Zhang YC, McCoy JP and Leonard WJ: The transcription factor GABP is a critical regulator of B lymphocyte development. Immunity 26: 421-431, 2007.

29. Yang ZF, Drumea K, Cormier J, Wang J, Zhu X and Rosmarin AG: GABP transcription factor is required for myeloid differentiation, in part, through its control of Gfi-1 expression. Blood 118: 2243-2253, 2011.

30. Manukjan G, Ripperger T, Santer L, von Neuhoff N, Ganser A, Schambach A, Schlegelberger B and Steinemann D: Expression of the ETS transcription factor GABP $\alpha$ is positively correlated to the BCR-ABL1/ABL1 ratio in CML patients and affects imatinib sensitivity in vitro. Exp Hematol 43: 880-890, 2015.
31. Yang ZF, Mott S and Rosmarin AG: The Ets transcription factor GABP is required for cell-cycle progression. Nat Cell Biol 9: 339-346, 2007

32. Wu H, Xiao Y, Zhang S, Ji S, Wei L, Fan F, Geng J, Tian J, Sun X, Qin F, et al: The Ets transcription factor GABP is a component of the hippo pathway essential for growth and antioxidant defense. Cell Rep 3: 1663-1677, 2013.

33. Castelo-Branco P, Choufani S, Mack S, Gallagher D, Zhang C, Lipman T, Zhukova N, Walker EJ, Martin D, Merino D, et al: Methylation of the TERT promoter and risk stratification of childhood brain tumours: An integrative genomic and molecular study. Lancet Oncol 14: 534-542, 2013.

34. Shay JW and Bacchetti S: A survey of telomerase activity in human cancer. Eur J Cancer 33: 787-791, 1997.

35. Killela PJ, Reitman ZJ, Jiao Y, Bettegowda C, Agrawal N, Diaz LA Jr, Friedman AH, Friedman H, Gallia GL, Giovanella BC, et al: TERT promoter mutations occur frequently in gliomas and a subset of tumors derived from cells with low rates of self-renewal. Proc Natl Acad Sci USA 110: 6021-6026, 2013.

36. Bell RJ, Rube HT, Kreig A, Mancini A, Fouse SD, Nagarajan RP, Choi S, Hong C, He D, Pekmezci M, et al: Cancer. The transcription factor GABP selectively binds and activates the mutant TERT promoter in cancer. Science 348: 1036-1039, 2015.

37. Shapiro GI: Cyclin-dependent kinase pathways as targets for cancer treatment. J Clin Oncol 24: 1770-1783, 2006.

38. Abbas T and Dutta A: p21 in cancer: Intricate networks and multiple activities. Nat Rev Cancer 9: 400-414, 2009.

39. Cazzalini O, Scovassi AI, Savio M, Stivala LA and Prosperi E: Multiple roles of the cell cycle inhibitor p21(CDKN1A) in the DNA damage response. Mutat Res 704: 12-20, 2010.

40. Ritter HD, Antonova L and Mueller CR: The unliganded glucocorticoid receptor positively regulates the tumor suppressor gene BRCA1 through GABP beta. Mol Cancer Res 10: 558-569, 2012.

41. Somasundaram K, Zhang H, Zeng YX, Houvras Y, Peng Y, Zhang H, Wu GS, Licht JD, Weber BL and El-Deiry WS: Arrest of the cell cycle by the tumour-suppressor BRCA1 requires the CDK-inhibitor p21 ${ }^{\mathrm{WAF} 1 / \mathrm{CiP} 1}$. Nature 389: 187-190, 1997.

42. Murai M and Oya M: Renal cell carcinoma: Etiology, incidence and epidemiology. Curr Opin Urol 14: 229-233, 2004.

43. Rini BI, Rathmell WK and Godley P: Renal cell carcinoma. Curr Opin Oncol 20: 300-306, 2008.

44. Rini BI: Stabilization of disease in patients with metastatic renal cell carcinoma using sorafenib. Nat Clin Pract Oncol 3: 602-603, 2006.

45. Motzer RJ, Bacik J, Schwartz LH, Reuter V, Russo P, Marion S and Mazumdar M: Prognostic factors for survival in previously treated patients with metastatic renal cell carcinoma. J Clin Oncol 22: 454-463, 2004

46. Eccles SA: Heparanase: Breaking down barriers in tumors. Nat Med 5: 735-736, 1999.

47. Engbring JA and Kleinman HK: The basement membrane matrix in malignancy. J Pathol 200: 465-470, 2003.

48. Vlodavsky I, Beckhove P, Lerner I, Pisano C, Meirovitz A, Ilan N and Elkin M: Significance of heparanase in cancer and inflammation. Cancer Microenviron 5: 115-132, 2012.

49. Jiang P, Kumar A, Parrillo JE, Dempsey LA, Platt JL, Prinz RA and $\mathrm{Xu} \mathrm{X}$ : Cloning and characterization of the human heparanase-1 (HPR1) gene promoter: Role of GA-binding protein and Sp1 in regulating HPR1 basal promoter activity. J Biol Chem 277: 8989-8998, 2002.

50. Rao G, Liu D, Xing M, Tauler J, Prinz RA and Xu X: Induction of heparanase-1 expression by mutant B-Raf kinase: Role of GA binding protein in heparanase-1 promoter activation. Neoplasia 12: 946-956, 2010. 